\title{
Keine großen Überraschungen, aber interessante Anregungen: Das Forschungsgutachten zur Psychotherapieausbildung ist da
}

\author{
Winfried Rief \\ Fachbereich Psychologie, Philipps-Universität Marburg, Deutschland
}

Von vielen mit Spannung erwartet wurde Anfang Mai 2009 das Forschungsgutachten zur Psychotherapieausbildung veröffentlicht. Im Auftrag des Bundesministeriums für Gesundheit wurde es von der Gutachtergruppe um Prof. Dr. Bernhard Strauß erstellt [Strauß et al., 2009], um die Ausbildungssituation von Psychologischen Psychotherapeuten ${ }^{1}$ und Kinder- und Jugendpsychotherapeuten (KJP) zu beleuchten. Dabei legt die Gutachtergruppe einen vom Umfang und Inhalt beeindruckenden Bericht vor. Das Gesamtwerk hat über 400 Seiten, hinzu kommen zahlreiche Anhänge und Detailinformationen. Alle 16 Landesprüfungsämter wurden per Fragebogen befragt, zusätzlich 184 Kliniken, 89 Ausbildungspraxen, 129 Ausbildungsinstitutsleiter, 2200 Lehrkräfte (Kursleiter, Supervisoren, Selbsterfahrungsleiter) und 480 Psychologiestudierende (jeweils Zahl der antwortenden Personen). Außerdem wurden 66 Experten im Rahmen einer Delphi-Befragung einbezogen. Über 11000 Psychotherapeuten wurden angeschrieben, von denen 3223 auswertbare Fragebögen zurücksandten. In Berlin fand ein Hearing mit über 100 Fachverbänden statt. Es ist kaum vorstellbar, wie die Gutachtergruppe diese Daten innerhalb von 1 Jahr und 4 Monaten sammeln, aufbereiten und systematisch auswerten konnte. Hierfür gebührt ihr höchste Anerkennung. Der Hauptanlass für das Gutachten ist die Notwendigkeit, das Psychotherapeutengesetz (PsychThG) an die neuen Studiengänge mit Bachelor- und Masterabschluss anzupassen; bisher ist es nur an den Diplomabschlüssen orientiert. Nachfolgend werden einige Einzelergebnisse dargestellt, Probleme und Kritikpunkte geäußert sowie die Veränderungsvorschläge der Gutachtergruppe diskutiert.

${ }^{1}$ Aus Gründen der Lesbarkeit wird nur die männliche Form verwendet, dennoch sind grundsätzlich beide Geschlechter angesprochen.

\section{KARGER}

Fax +497614520714

Information@Karger.de

www.karger.com (c) 2009 S. Karger GmbH, Freiburg

Accessible online at:

www.karger.com/ver

\section{Einige Ergebnisse aus dem Forschungsgutachten}

Die meisten Befragten sind mit vielen Regelungen des PsychThG und der angeschlossenen Ausbildungs- und Prüfungsverordnung APrV zufrieden. Offensichtlich haben sich viele Regelungen des PsychThG bewährt, und die psychologischen Psychotherapeuten und Kinder- und Jugendpsychotherapeuten wurden fest im Gesundheitssystem verankert. Gerade die praktische Ausbildung (Fallarbeit) mit der dazugehörenden Supervision wird sehr positiv eingeschätzt; es gibt kaum einen Ausbildungsinhalt, der nicht überwiegend $\mathrm{Zu}$ stimmung erfährt. Die Mehrzahl findet weiterhin eine Orientierung an übergeordneten Psychotherapieverfahren für die Ausbildung sinnvoll (bei verhaltenstherapeutischer Orientierung: $67 \%$ Befürwortung; bei psychodynamisch/psychoanalytischer Orientierung: $80 \%$ Befürwortung). Die durchschnittliche Ausbildungsdauer ist wie befürchtet länger als vorgesehen (3,94 Jahre bei Vollzeit-, 5,41 bei Teilzeitausbildung). Leider problematisiert das Gutachten kaum, dass dadurch die meisten Ausbildungsteilnehmer deutlich über 30 Jahre alt sind, wenn sie die Approbation erhalten. Die Konsequenzen für Familienplanung, Stellensicherheit oder adäquate Entlohnung sind Problemfacetten, die noch kritischer beleuchtet werden müssen. Insbesondere für den wissenschaftlichen Nachwuchs ist es kaum tragbar, 4-5 Jahre mit der Psychotherapieausbildung und 3-5 Jahre mit der Promotion zu verbringen, bevor die eigentliche wissenschaftliche Laufbahn beginnen kann. Hier sind Sonderregelungen notwendig, die durch Kombinationsmöglichkeiten, erleichterte Anerkennung von Leistungen, modifizierte Ansprüche an die Praxiszeit etc. eine internationale Angleichung ermöglichen.

Gibt es genügend psychotherapeutischen Nachwuchs? Eine Befragung der Psychologiestudierenden ergab, dass sehr großes Interesse an einer Psychotherapieausbildung besteht. Bereits während des Studiums sind 25\% definitiv entschieden 
und weitere $29 \%$ ziemlich entschieden, eine Psychotherapieausbildung zu beginnen (38\% Verhaltenstherapie vs. 19,6\% Tiefenpsychologie/Psychoanalyse bei der befragten Stichprobe). Hauptentscheidungsgrund für ein bestimmtes Psychotherapieverfahren sind das vertretene Menschenbild und die praktizierten therapeutischen Stile. Allerdings zögern auch 4 von 5 Psychologiestudierende wegen der finanziellen Belastung, postgradual eine Psychotherapieausbildung zu beginnen. Trotzdem ist das Interesse, im klinischen Bereich tätig zu werden und nach dem Studium eine Psychotherapieausbildung durchzuführen, ungebrochen hoch.

Im Vorfeld immer wieder heiß diskutiert war die Frage nach dem «Psychiatriejahr» (genauer: praktische Tätigkeit) [vgl. Hohagen und Rief, 2009]. Ziel der Regelung zum Psychiatriejahr war, dass die Ausbildungsteilnehmer Erfahrungen mit einem breiten Diagnosespektrum machen und während der Ausbildung dabei gute fachliche Anleitung erhalten. Die meisten Ausbildungsteilnehmer werden jedoch während ihres Psychiatriejahres zur selbständigen Durchführung von Einzeltherapie (74\%) bzw. Gruppentherapien (65\%) eingesetzt, so dass sie primär Dienstleistungen für den Krankenhausträger erbringen. Erschreckend ist, dass 38\% der Befragten angeben, dass sie während des Psychiatriejahres eigene Arbeitsbereiche übernehmen mussten, ohne fachliche Anleitung erhalten zu haben. Demnach wird mehr als jeder Dritte «ins kalte Wasser geworfen» und führt - zumindest subjektiv - ohne fachliche Anleitung Therapien durch. Aber auch bezüglich der Zielsetzung, dass während der Psychiatriezeit ein breites Spektrum an Krankheitsbildern/Diagnosen kennengelernt werden soll, kommen Zweifel auf. Die Diagnosestatistiken der behandelten Fälle während der Psychiatriezeit unterscheiden sich laut Forschungsgutachten nur marginal von den Diagnosestatistiken der behandelten ambulanten Fälle während der praktischen Tätigkeit in der Ausbildungsambulanz. Kenntnisse über die diagnostische Breite werden also bisher nicht durch das Psychiatriejahr erreicht.

Ein bedenkliches Ergebnis der Erhebungen ist, dass viele Ausbildungskandidaten während der Psychiatriezeit mit wesentlichen Krankheitsbildern keine Erfahrungen sammeln. So geben $43 \%$ der Absolventen an, während der Psychiatriezeit keine Erfahrungen mit Patienten mit Suchterkrankungen gewonnen zu haben, $40 \%$ haben keine Erfahrungen mit schizophren Erkrankten gesammelt, $48 \%$ haben keine Erfahrungen mit Essstörungen gesammelt, 3 von 4 haben keine Erfahrungen mit psychischen Problemen bei geistiger Behinderung machen können, und 84\% haben keine Erfahrungen mit Personen mit sexuellen Funktionsstörungen gemacht. Erwartungsgemäß fällt auch der Bereich Psychosomatik (psychische Störungen bei körperlichen Krankheiten) vergleichbar problematisch aus, in dem während des Psychiatriejahres zu 73\% keine Erfahrungen gesammelt werden. Obwohl zum Beispiel mehr als die Hälfte aller Personen mit kardiovaskulären oder muskuloskelettalen Erkrankungen psychische Erkrankungen aufweist [Baumeister, 2004], sammeln viele Ausbildungsteil- nehmer während der praktischen Tätigkeit für diese medizinisch-psychologischen Mischprobleme zu wenig Erfahrungen. Da die Ziele der praktischen Tätigkeit offensichtlich nicht erreicht werden, überrascht es wenig, dass sich mehr als die Hälfte der befragten Experten für eine Verkürzung des Psychiatriejahres ausspricht. Die Problematik wird dadurch unterstrichen, dass 37\% während des Psychiatriejahres keinerlei Einkommen erhalten (aber die Psychotherapieausbildung bezahlen müssen) und weitere $10 \%$ mit einem Einkommen von unter 500 EUR deutlich unter dem Existenzminimum liegen. Noch schwieriger ist die Lebenssituation während des Psychiatriejahres für diejenigen, die die Ausbildung in Kinder- und Jugendpsychotherapie machen wollen, da es in diesem Bereich noch seltener Ausbildungsplätze mit Bezahlung gibt.

Bezüglich der Kostenstruktur kritisiert die Gutachtergruppe zu Recht, dass diese hoch intransparent ist und es Ausbildungskandidaten kaum möglich ist, die Finanzierungsstruktur verschiedener Ausbildungsinstitute zu vergleichen. Am einfachsten gelingt dies noch bei den realen Kosten, die die theoretische Ausbildung, die praktische Ausbildung, die Supervision und die Selbsterfahrung umfassen. Hier sind die universitär gebundenen Ausbildungsinstitute mit im Mittel ca. 17000 EUR Kosten deutlich günstiger als die privaten Institute mit 33400 EUR. Allerdings weisen auch einige private Institute gerade für die Ausbildung in Verhaltenstherapie ähnliche Kostenstrukturen auf wie die universitären Institute. Wird der Großteil der geforderten Ausbildungstherapien (praktische Ausbildung) am Ausbildungsinstitut absolviert und schüttet dieses einen entsprechenden Betrag an die Ausbildungsteilnehmer aus, so kann diese Rückvergütung die Höhe der oben beschriebenen Kosten erreichen, so dass die Rechnung zumindest vermeintlich «Null auf Null» ausgeht. Dieses Aufrechnungsmodell (im Gutachten: «Netto-Kosten») haben die Gutachter jedoch meines Erachtens voreilig übernommen. Eine korrekte ökonomische Analyse muss berücksichtigen, dass während der Ausbildungszeit erniedrigte Einkommensverhältnisse bestehen und deshalb die Ausbildungsdauer sowie die geforderten Leistungen mit einzubeziehen sind. Dauert eine Ausbildung zum Beispiel 7 statt 3 Jahre, so hat der Ausbildungskandidat die Differenz von 4 Jahren zu tragen, in denen er noch nicht das volle Gehalt eines Approbierten erhalten wird. Gleiches gilt, wenn im einen Fall die Ausbildungskosten dadurch refinanziert werden, dass 600 Ausbildungstherapie-Stunden absolviert werden, im anderen Fall (z.B. kombinierte Psychoanalyse-/Tiefenpsychologie) durch 1400 Ausbildungstherapie-Stunden eine Refinanzierung erfolgen muss, also indem ein Teilnehmer viele Monate länger umsonst arbeitet. Hier wären echte ökonomische Analysen wichtig, und es ist bedauerlich, dass die Gutachter an dieser Stelle eher zur Verschleierung als zur Transparenz der Kostenstrukturen beitragen.

Eine der Hauptfragen, die das Gutachten beantworten soll, betrifft die Zugangsvoraussetzungen zur Psychotherapieausbildung. So können die Ausbildung in Psychologischer 
Psychotherapie bisher nur Personen mit einem Diplom in Psychologie beginnen, während im Bereich Kinder- und Jugendpsychotherapie auch Sozialpädagogen, Diplompädagogen und andere Berufe zugelassen werden, zum Teil mit nur 3-jährigem Hochschulstudium. Hier hat sich die weit überwiegende Mehrheit aller Experten klar dafür ausgesprochen, dass ein Master die Voraussetzung für eine Psychotherapieausbildung darstellen muss. Auch im europäischen Ausland reicht der Bachelor in der Regel nicht für die Zulassung zur Psychotherapieausbildung. Die befragten Ärzte, die im Rahmen der Kliniktätigkeit oder von Schulungen mit vielen Ausbildungskandidaten zu tun haben, haben sich zu 100\% für den Master als Zugangsvoraussetzung ausgesprochen.

\section{Probleme bei der Interpretation des Forschungs- gutachtens}

Jede Studie hat ihre Begrenzungen, die bei der Interpretation der Ergebnisse berücksichtigt werden müssen. Deshalb sollen hier auch Kritikpunkte genannt werden, die jedoch die außerordentliche Leistung der Gutachtergruppe in keiner Weise limitieren sollen.

Wie häufig bei solchen Erhebungen gibt es auch bei dieser substanzielle Selektionsprobleme. Zum Teil haben nur 20\% der angeschriebenen Zielgruppe geantwortet, so dass eine Hochrechnung auf die Gesamtheit von $100 \%$ mit einer erheblichen Fehlerwahrscheinlichkeit verbunden ist. Des Weiteren werden immer wieder Gruppenunterschiede berechnet (z.B. zwischen Verhaltenstherapeuten und Tiefenpsychologen), die in Zukunft sicherlich zu Fehlinterpretationen Anlass geben werden. Dabei wird nämlich nicht berücksichtigt, dass die Teilnehmer nicht randomisiert auf die verschiedenen Ausbildungsgänge aufgeteilt werden, sondern gezielt nach persönlichen Kriterien ein Psychotherapieverfahren auswählen. Dies bedeutet, dass es von vornherein Stichprobenunterschiede gibt, die aber nicht bekannt sind. Des Weiteren hat sich in den letzten Jahren eine deutliche Verlagerung zugunsten der Verhaltenstherapie-Ausbildungsinstitute ergeben, so dass hier die Kohorten größer sind und die Organisation höhere Herausforderungen stellt. Das Forschungsgutachten stellt dazu lapidar fest, dass die Organisationsbewertung für Ausbildungsinstitute «Tiefenpsychologie/Psychoanalyse» etwas besser ausfällt als für Ausbildungsinstitute «Verhaltenstherapie», ohne auf die zuvor genannten Unterschiede einzugehen. Auch sind die nominellen Unterschiede zwischen den Verfahren in den meisten Befragungen gering, wenn auch manchmal wegen der großen Stichprobe signifikant. Aus diesen Gründen können diese Gruppenvergleiche meines Erachtens zwar für persönliche Überlegungen herangezogen werden, sollten jedoch nicht im größeren Stile interpretiert werden.

Überhaupt erscheint es etwas willkürlich, wann das Gutachten welche Unterschiede als relevant interpretiert. An manchen Stellen wird betont, dass Änderungsbedarf besteht, weil $20 \%$ mit einer Regelung unzufrieden sind; auf der anderen Seite werden bestimmte Aspekte als gut gelöst bewertet, weil $80 \%$ damit zufrieden sind. In beiden Fällen ist jedoch die Antwortverteilung identisch, so dass eine unterschiedliche Interpretation begründet werden müsste.

Bezüglich der Kostenstruktur bringt das Forschungsgutachten nur begrenzt Licht in den Dschungel der Gebührenordnungen. Zwar wird korrekt festgehalten, dass eine Verhaltenstherapie-Ausbildung deutlich günstiger ist als eine Ausbildung in psychodynamischen oder psychoanalytischen Verfahren, die Berechnung von «Nettokosten» führt jedoch zu dubiosen Ergebnissen. Eine echte ökonomische Analyse, auch unter gesellschaftlicher Perspektive wäre interessant. Last but not least wird die schwierige Situation des wissenschaftlichen Nachwuchses im Bereich Psychotherapieforschung zu wenig beleuchtet. Psychotherapieforscher in Deutschland haben einen erheblichen Standortnachteil, da Psychotherapieausbildung und Promotion zu wenig kombiniert sind und häufig nur nacheinander absolviert werden können, was zu enormen Ausbildungsverlängerungen führt.

\section{Kritische Kommentierung der Vorschläge der Gutachter}

Die Vorschläge der Gutachterkommission, in welchen Bereichen die Regelungen des PsychThG und der APrV geändert werden sollten, werden nachfolgend vorgestellt und kommentiert.

a) Zugang zur Psychotherapieausbildung sollen Personen erhalten, die ein sozialwissenschaftliches Studium mit dem Master abgeschlossen haben. Es ist zu hoffen, dass die Gutachter dabei auch die naturwissenschaftlichen Psychologiestudiengänge einschließen wollen. Der Studienabschluss muss 85 Credit Points (CP) in allgemeinen Inhalten der Psychologie enthalten sowie zusätzlich 30 CPs in Klinischer Psychologie, 10 CPs durch Praktika und 25 CPs durch eine Masterarbeit mit psychologischem Bezug. Dadurch wird der zu erwartenden Heterogenität von Hochschulstudiengängen und deren Bezeichnungen Rechnung getragen.

Auf den ersten Blick fiele somit das Monopol des Psychologiestudiums als Zugang zur Psychotherapieausbildung für Psychologische Psychotherapeuten. Allerdings ist durch die Diversifizierung dieses Problem kaum anders zu regeln, als psychologische Ausbildungsinhalte zu fordern, nicht psychologische Ausbildungstitel. Gerade die eindeutige Festlegung auf einen Master-Abschluss im Gutachten ist hier hilfreich und bestens begründet. Allerdings werden einige Hochschulinstitute Schwierigkeiten mit den Forderungen an Studieninhalte bekommen. So fordern die Gutachter für psychologische Grundlagenfächer mindestens 5 CPs (Vorschlag der DGPs für Bachelor-Studiengänge: $4 \mathrm{CPs}$ ) und eine besondere Vertiefung in Entwicklungspsychologie (10 CPs) und Pädagogischer Psychologie (5 CPs). Vermutlich standen die Vorbe- 
reitungen für den KJP-Bereich hier Pate, aber dies führt de facto zu einer unnötigen Einengung. Wenn eine Vertiefung im Anwendungsfach Pädagogische Psychologie als zweitem Anwendungsfach neben Klinischer Psychologie erzwungen wird, können Studierende sich nicht mehr in anderen wichtigen Vertiefungsbereichen qualifizieren (z.B. Neuropsychologie, Occupational Health aus der Arbeits- und Organisationspsychologie, Psychosomatik, Klinische Kinder- und Jugendpsychologie). An diesem Punkt wäre eine flexiblere Interpretation wichtig. So könnte man alternativ an der Forderung von 85 CPs aus dem nichtklinischen Bereich der Psychologie festhalten, dabei die Mindestanforderung pro Teilfach auf 4 CPs (außer: Methoden: 10, Allgemeine Psychologie: 8) festsetzen und ein Modul in einem nichtklinischen Anwendungsfach fordern, ohne dieses zu spezifizieren.

Andere Studiengänge, wie zum Beispiel die Pädagogik, können nach diesem Vorschlag ebenfalls die Voraussetzungen auch zum Erwachsenenpsychotherapeuten bieten, allerdings wird eine starke Psychologisierung dieser Studiengänge gefordert. Hier ergibt sich ein Unterschied zu den Vorschlägen der Bundespsychotherapeutenkammer und der DGPs. Dieser Alternativvorschlag baut weiterhin auf psychologische Psychotherapeuten versus Kinder- und Jugendpsychotherapeuten auf, lässt Pädagogik-Absolventen nur für die KJP-Ausbildung zu, belässt den Pädagogik-Studiengängen jedoch mehr Individualität und erzwingt weniger Psychologisierung. Welcher Ansatz sinnvoller ist, müssen weitere Diskussionen zeigen. Falls die Voraussetzungen für eine Psychotherapieausbildung durch das Studium noch nicht vollständig erfüllt sind, schlägt die Gutachtergruppe die Möglichkeit eines Propädeutikums (bis zu 35 CPs) vor. Dieses kann an Hochschuleinrichtungen oder nach deren Vorschlägen auch bei den Ausbildungsinstituten erfolgen. An dieser Stelle ist der Vorschlag noch wenig durchdacht. Welche Ausbildungsinhalte werden gehäuft fehlen, vor allem bei Personen mit nichtpsychologischen (Fach-) Hochschulabschlüssen? Vermutlich Methodenkenntnisse, Kenntnisse in Grundlagen der Psychologie, diagnostische Methoden. Sollen gerade diese Kerninhalte eines wissenschaftlichen Studiums von privaten Ausbildungsinstituten angeboten werden? Es ist unwahrscheinlich, dass private Institute gerade diese in der nötigen Qualität anbieten. Eine Verpflichtung, solche Ausbildungsinhalte durch qualifizierte Hochschullehrer anzubieten, ist notwendig.

b) Duales System beibehalten, jedoch Möglichkeit, in begrenzter Form Modellstudiengänge auszurichten: Die Gutachtergruppe spricht sich insgesamt für die Fortsetzung des dualen Systems aus (Ausbildung in Psychotherapie nach Ausbildung in einem Zugangsberuf). Allerdings weist die Gutachtergruppe auch auf Möglichkeiten zur Einrichtung von Modellstudiengängen hin. Gerade in der Medizin wurden damit sehr gute Erfahrungen gemacht. Für die Psychotherapie werden dringend Erfahrungen benötigt, wie Modellstudiengänge die Masterausbildung, gegebenenfalls anschließende Promotion und Psychotherapieausbildung integrieren kön- nen. Auch dafür muss der Gesetzgeber die entsprechenden Voraussetzungen schaffen (z.B. Öffnungsklauseln).

c) Erwachsenen-Psychotherapie versus Kinder- und Jugendpsychotherapie als gleichberechtigte Berufe. Hier favorisieren die Gutachter eine Lösung, die einen vorgeschalteten «Common Trunk» von Inhalten vorsieht, die bei Kindern und Erwachsenen in der Psychotherapie gleich sind. Erst nachfolgend soll eine Differenzierung erfolgen, bei der sich die Ausbildungskandidaten auf die Schwerpunkte Kinder und Jugendliche oder Erwachsene aufteilen. Dies klingt zunächst vernünftig, dürfte aber in der Praxis nicht sinnvoll durchzuführen sein. Was ist der Common Trunk zwischen KJP und Erwachsenenpsychotherapie, z.B. bei Angststörungen? Es sind die Störungsmodelle und theoretischen Hintergründe, während das praktische Vorgehen (z.B. Expositionsvorbereitung bei Kindern vs. Erwachsenen) sich deutlich unterscheidet. Dies birgt die Gefahr, dass der Common Trunk in hohem Maße theorielastig wird und innerhalb der Psychotherapieausbildung dasselbe Thema bei der spezifizierten Vertiefung wieder aufgegriffen werden muss, nun aber auf eine Altersgruppe bezogen wird. Damit sind doppelte und dreifache Darbietungen (Studium, Common Trunk, Vertiefung) von theoretischen Inhalten programmiert.

d) Lobenswert ist die Betonung, dass Leistungen z.B. auch aus dem grundständigen Studium anerkannt werden sollen. Dies betrifft z.B. Praktika. In manchen Studiengängen wird bereits während der Bachelor- und Masterzeit ein Praktikum von 6 Monaten vorgesehen. Trotzdem mussten diese Studierenden bisher für die Psychiatriezeit und die praktische Tätigkeit der Psychotherapieausbildung im Allgemeinen genauso viel Zeit investieren wie Personen, die während des Studiums kein halbjähriges Psychiatriepraktikum gemacht hatten. Hier ist eine Flexibilisierung notwendig und sinnvoll.

e) Eine Verkürzung der Psychotherapieausbildung, z.B. durch Verkürzung der praktischen Tätigkeit von 1800 auf 1200 h sowie Reduktion der «freien Spitze» ist sehr zu begrüßen.

f) Optionale Kompetenzerweiterung nach entsprechender Schulung der Ausbildungskandidaten, so dass diese auch Arbeitsunfähigkeitsbescheinigungen, Überweisungen zu entsprechenden Fachärzten (z.B. Rücküberweisung zum Psychiater) und Einweisungen in psychiatrische und psychotherapeutische Einrichtungen vornehmen können. Auch eine Verordnung psychotherapierelevanter Heil- und Hilfsmittel wird vorgeschlagen. Mit diesem Vorschlag wird der Tatsache Rechnung getragen, dass Psychotherapeuten in der Gesundheitsversorgung oftmals diejenigen sind, die den entsprechenden Patienten am häufigsten sehen und am meisten über seine Lebensbedingungen wissen. Eine Option zur Verschreibung von Psychopharmaka bei entsprechender Zusatzqualifikation sowie zur Anordnung von Zwangseinweisungen wird jedoch von der Gutachtergruppe nicht empfohlen.

g) Erhöhung des Selbsterfahrungsteils auf $150 \mathrm{~h}$, davon $50 \mathrm{~h}$ Einzelselbsterfahrung: Selbsterfahrung wird gerade in der tiefenpsychologischen und psychoanalytischen Ausbildung 
sehr hoch geschätzt, und auch die Gruppenselbsterfahrung bei der Ausbildung in Verhaltenstherapie bekam gute Noten. Einzelselbsterfahrung ist in der Verhaltenstherapie noch selten, wurde jedoch ebenfalls positiv bewertet. Allerdings ist dies kein Grund, diese deutlich auszubauen und den Bereich Selbsterfahrung insgesamt zu erhöhen. Gerade in der Verhaltenstherapieausbildung wird offensichtlich die Einzelsupervision deutlich besser eingeschätzt als die Selbsterfahrung. Wegen des hohen Patientendurchlaufs in der Ausbildung von Verhaltenstherapeuten ist diese auch ganz besonders wichtig. Demgegenüber gibt es für Einzelselbsterfahrung in der Verhaltenstherapie nicht einmal einen theoretischen Rahmen, geschweige denn eine Vorstellung, wie diese die Qualität der durchgeführten Psychotherapie verbessern soll. Manche wichtigen Inhalte werden also in der einen Ausbildung eher im Rahmen der Selbsterfahrung, in der anderen Ausbildung eher im Rahmen der Einzelsupervision thematisiert. In keinem Bereich gibt es wissenschaftliche Belege, dass Selbsterfahrung zu besserer Psychotherapie führt [Rief et al., 2009], wenn auch manche Klinikerurteile dies nahelegen. Bevor diese Fragen nicht klar beantwortet sind, ist eine Ausweitung der Selbsterfahrung nicht sinnvoll. Deshalb führt dieser Vorschlag der Gutachtergruppe nur zu einer unbegründeten Verteuerung und Verlängerung der Ausbildung. Nicht nachvollziehbar ist in diesem Kontext, dass die Gutachtergruppe eine Reduktion der verpflichtenden Einzelsupervision auf 25 der 150 geforderten Stunden vorschlägt (bisher: 50). Einzelsupervision wurde neben der praktischen Tätigkeit am besten bewertet, in der Regel sogar besser als Einzelselbsterfahrung! Deshalb wäre es besser, als Gesamtforderung zu definieren, dass Supervision und Selbsterfahrung zusammen $270 \mathrm{~h}$ umfassen sollen (wie bisher), jedoch je nach Curriculum und Schwerpunkt mehr Supervision oder mehr Selbsterfahrung beinhalten können (gegebenenfalls Mindestforderung pro Baustein $100 \mathrm{~h}$ ). Dabei kann festgelegt werden, dass mindestens $50 \mathrm{~h}$ im Einzelformat erfolgen müssen, wobei offen bleiben kann, ob dies eher Selbsterfahrung oder eher die Supervision betrifft.

h) Reduktion der verpflichtenden Psychiatriezeit von 1200 auf 600 h. Dieser Vorschlag wird noch zu einigen Diskussionen Anlass geben und die DGPPN hat entsprechend kritische Kommentare dazu vorbereitet (siehe Pressemitteilung vom 25.06.2009, www.dgppn.de). Die positiven Zielsetzungen, die mit der Einführung des Psychiatriejahres verbunden waren, sind jedoch offensichtlich nicht erfüllt worden. Weder die Anleitung ist zufriedenstellend noch wird eine entsprechende Störungsbreite kennengelernt. Vielmehr müssen die Ausbildungsteilnehmer unter schlechten sozialen Bedingungen reguläre Dienstleistungen selbständig erbringen. Deshalb besteht definitiv Änderungsbedarf, zum einen in Richtung einer Qualitätssteigerung (z.B. Sicherstellung eines entsprechenden Diagnosespektrums, mit dem Erfahrungen gesammelt werden; Sicherstellung von fachkompetenter Einarbeitung usw.), zum anderen muss bei der bestehenden schlechten sozialen Absicherung der Stellenwert des Psychiatriejahrs deutlich reduziert werden. Eine Festigung oder gar Ausweitung der Regelungen zum Psychiatriejahr hat keinen Sinn, wenn nicht auch die Finanzierungsregelungen für alle Seiten befriedigend geklärt werden. Eine Bafög-Regelung, wie von der Gutachtergruppe andiskutiert, für die Zusatzqualifikation von Personen, die bereits einen akademischen Abschluss haben, halte ich für indiskutabel. Wird keine adäquate Finanzierungsregelung erreicht, gibt es auch im Sinne einer Verkürzung der Gesamtausbildung nur den Weg über eine verkürzte Zeit, die in der Psychiatrie verbracht werden muss. Bei entsprechender Organisation sollte das geforderte Ziel, ein breites Störungsspektrum kennenzulernen, auch in kürzerere Zeit (z.B. 6 Monaten) erreicht werden.

\section{Fazit}

Die Erhebungen im Rahmen des Forschungsgutachtens zeigen in den meisten Bereichen eine hohe Zufriedenheit mit den bestehenden Regelungen zur Psychotherapieausbildung. Die meisten Ausbildungsinhalte werden ausgesprochen positiv bewertet. Über 99\% der antwortenden Ausbildungsteilnehmer sind auch mit der Ausbildungsrichtung zufrieden, die sie gewählt haben. Der Vorschlag, als Zugangsvoraussetzung zur Psychotherapieausbildung eine Hochschulausbildung mit Masterabschluss zu fordern, die bestimmte psychologische Inhalte abdeckt, ist im Gutachten gut begründet und kann eine gute Orientierungshilfe für Revisionen des PsychThG sein. Allerdings müssen an einigen Stellen auch Nachkorrekturen der Empfehlungen des Forschungsgutachtens erfolgen. Auch die besondere Situation des wissenschaftlichen Nachwuchses in Klinischer Psychologie wird bisher im Gutachten nicht berücksichtigt, ist aber für die Zukunft sehr bedeutsam, da durch diesen die Studierenden ausgebildet werden. Nichtsdestotrotz gebühren den Autorinnen und Autoren unser Dank und unsere Anerkennung für die immense geleistete Arbeit.

\section{Literatur}

Baumeister H, Höfler M, Jacobi F, Wittchen HU, Bengel J: Psychische Störungen

bei Patienten mit muskuloskelettalen und kardiovaskulären Erkrankungen im Vergleich zur Allgemeinbevölkerung. Z Klin Psychol Psychoth 2004;33:33-41.

Hohagen F, Rief W: Das Psychiatriejahr in der Ausbildung zum Psychologischen Psychotherapeuten. Verhaltenstherapie 2009;19:187-190.

Rief W, Freyberger HJ, Sartory G: Macht Selbsterfahrung bessere Therapeuten? Verhaltenstherapie 2009;19:53-57.

Strauß B, Barnow S, Brähler E, Fegert J, Fliegel S, Freyberger HJ, Goldberg L, Leuzinger-Bohleber M, Willutzki U: Forschungsgutachten zur Ausbildung von Psychologischen PsychotehrapeutInnen und Kinder- und JugendpsychotherapeutInnen. Im Auftrag des Bundesministeriums für Gesundheit. www.bmg.bund.de 